\title{
Escala de Pensamento Existencial: análise Rasch da versão Portuguesa
}

\author{
The Scale for Existential Thinking: Rasch analysis of the Portuguese version
}

\author{
José Pacheco Miguel, José Tomás da Silva, Teresa Sousa Machado \\ Faculdade de Psicologia e Ciências da Educação - Universidade de Coimbra - Portugal
}

\begin{abstract}
Resumo
O presente estudo exploratório analisa as propriedades psicométricas da Scale for Existential Thinking (SET) numa amostra de universitários portugueses, usando a modelação Rasch. Os resultados indicam que os itens ajustam a uma estrutura latente unidimensional, pressuposto da modelação Rasch. A escala de resposta apresentou funcionamento adequado, permitindo que os parâmetros de pessoas e itens fossem estimados com elevada precisão. Análises DIF confirmaram a inexistência de diferenças nos resultados relativas ao género. Análises de correlação com medidas adicionais oferecem evidências de validade de construto e de critério. Discutem-se as limitações do estudo e as implicações psicométricas dos resultados para futuras investigações.

Palavras chave: pensamento existencial, Rasch, rating scale model, DIF
\end{abstract}

\begin{abstract}
The present study analyzes the psychometric properties of the Scale for Existential Thinking (SET) in a sample of Portuguese university students with the Rasch model. The results indicate that the items are well fitted to a latent unidimensional structure, as required by Rasch modeling. The response scale showed proper functioning; therefore, people and item parameters were estimated with high precision. DIF analyses confirmed no differences in the results concerning gender. Correlational analyses with additional measures support construct and criterion-related validity. Limitations of this study and the psychometric implications of its results are discussed, providing suggestions for future research.
\end{abstract}

Keywords: existential thinking, Rasch, rating scale model, DIF

As preocupações com as questões fundamentais da existência, tais como, o sentido da vida ou aquilo que acontece após a morte, constituem uma experiência humana universal, ao ponto da maior parte das pessoas terem crenças formadas acerca das questões existenciais. Para além disso, a capacidade transcultural de considerar as questões existenciais e de procurar sentido para a própria existência, são dimensões importantes para o funcionamento humano ideal. No entanto, uma vez que as pessoas possuem capacidades distintas de exploração e compreensão das questões existenciais, diferindo de modo considerável na forma como ponderam estas questões nucleares, a psicologia necessita de instrumentos que permitam medir o pensamento existencial e avaliar os efeitos e correlatos deste construto.

Tendo em consideração esta necessidade, e com base na investigação sobre as múltiplas formas de inteligência da qual Gardner (2004) partiu para sugerir a inteligência existencial como um novo tipo de inteligência, Allan e Shearer (2012) propõem o construto pensamento existencial. Os autores definem este tipo de pensamento como a tendência para se envolver em preocupações fundamentais da existência e a capacidade do sujeito para realizar um processo de atribuição de sentido que o situe relativamente a estas questões existenciais. Segundo esta sua conceptualização, o pensamento existencial envolve principalmente um processo de construção de significado para atribuição de sentido à própria existência (Spinelli, 2005).

Partindo desta conceptualização, Shearer (2006) desenvolveu uma medida de auto-relato, a Scale for Existential Thinking (SET), cujo estudo psicométrico (dimensionalidade) proporcionou evidência que confirma a unidimensionalidade da medida com amostras mistas de adolescentes e adultos. Num estudo posterior, com uma amostra apenas de universitários de psicologia, Allan e Shearer (2012) confirmaram a unifactorialidade da escala e uma elevada consistência interna, esta medida com alfa de Cronbach. As correlações positivas e estatisticamente significante da SET com medidas adicionais de variáveis teoricamente afins (e.g., satisfação com a vida, procura e presença de sentido para a vida) constituem, segundo os autores, evidência confirmatória da validade convergente da escala.

Tendo em consideração as bons indicadores psicométricos desta medida de auto-relato usada para avaliar o nível de preocupação com as questões existenciais e a capacidade de lhes atribuir um sentido, bem como a ausência de uma escala eficaz para medir o construto de forma precisa na população portuguesa, optou-se por proceder à adaptação da SET. Este estudo exploratório visou avaliar as propriedades psicométricas (dimensionalidade e fidelidade) da versão portuguesa da escala numa amostra de jovens adultos universitários, no contexto da teoria de resposta ao item (TRI). Especificamente, optou-se pelo modelo de Rasch que, pelas suas pelas suas propriedades métricas ideais, usa apenas as respostas dos sujeitos enquanto estatísticas suficientes para avaliar o nível de dificuldade dos itens e o nível de atributo (pensamento existencial) nos sujeitos de forma simultânea e independente (Wright \& Mok, 2004).

Examinaram-se ainda as relações do pensamento existencial com variáveis teoricamente relacionadas com este construto, designadamente, a inteligência espiritual, o bem-estar subjectivo (afecto e sentido para a vida), a autoestima e a perspectiva temporal de futuro, enquanto indicadores de validade convergente. 


\section{Método}

Foram realizadas análises de Rasch, com o software Winsteps (Linacre, 2011), versão 3.73.0. Especificamente, dado o formato politómico invariante de todos os itens da escala, as estimativas dos parâmetros relativos aos itens, aos sujeitos e às categorias de resposta foram calculadas com o Rating Scale Model (RSM, Wright \& Masters, 1982). O RSM é uma extensão do modelo de Rasch para itens politómicos e, segundo Linacre (2002), a sua formalização matemática consiste na expressão:

$$
\log \left[P_{n i k} / P_{n i(k-1)}\right]=B_{n}-D_{i}-F_{k}
$$

$P_{\text {nik }}$ representa a probabilidade da pessoa $n$ responder na categoria $k$ do item $i, P_{n i(k-1)}$ é a probabilidade da pessoa $n$ responder na categoria $k-1$ do item $i, B_{n}$ é a medida da pessoa $n$ no traço avaliado, $D_{i}$ é a dificuldade do item $i$ e $F_{k}$ é a dificuldade do passo da categoria $k-1$ para a categoria $k$ (i.e., calibração do passo); esta calibração do passo $\left(F_{k}\right)$ é um limiar da escala de classificação definido como sendo a localização correspondente à equiprobabilidade de observação das categorias adjacentes $k-1$ e $k$.

A opção pelo RSM explica-se, para além das suas já referidas propriedades métricas que permitem que a comparação das pessoas seja independente dos itens usados para as medir e que a comparação dos itens seja invariante das pessoas utilizadas para os calibrar (objectividade específica), porque transforma os dados ordinais relativos às respostas dos sujeitos numa escala intervalar (Wright \& Mok, 2004). Em termos práticos, para além de apresentar estatísticas para o ajustamento da pessoa e do item, tem a vantagem de não necessitar de amostras grandes para estimar os seus parâmetros. Para além disso, permite ainda que se consiga determinar de forma empírica a qualidade discriminativa das categorias nas escalas de resposta de tipo Likert (Bond \& Fox, 2007).

Aavaliação da SET com o RSM centrou-se nos aspectos da validade de conteúdo, da validade estrutural e da validade substantiva propostos por Wolfe \& Smith (2007), com base no trabalho de Messick (1995).

\section{Participantes}

Participaram no estudo 200 alunos da Faculdade de Psicologia e de Ciências da Educação da Universidade de Coimbra. A amostra, não probabilística, tinha uma média de idades de $19.61(D P=1.94)$ anos e incluía 13\% $(n=$ $26)$ de alunos e $87 \%(n=174)$, maioritariamente $(82 \%, n$ = 164) sem histórico de insucesso académico. Não foi recolhida informação sobre a etnia por não ser uma variável relevante para os estudos realizados em Portugal (a maioria dos respondentes eram caucasianos europeus).

\section{Instrumentos}

As medidas usadas incluíram variáveis demográficas (sexo, idade, retenções escolares, nota da disciplina de filosofia no ensino secundário e habilitações académicas dos pais), a Scale for Existential Thinking, o Spiritual Intelligence Self-Report Inventory, a Positive and Negative Affect Schedule, a Rosenberg Self-Esteem Scale, a Future Time Perspective Scale e o Meaning in Life Questionnaire.
Scale for Existential Thinking (SET; Allan \& Shearer, 2012). É uma medida unidimensional de auto-relato do pensamento existencial incluindo 11 itens $(\alpha=.93)$ cuja soma permite calcular um resultado global a variar entre 11 e 55. Os participantes avaliam a frequência com que se envolvem em vários comportamentos de pensamento existencial numa escala com 6 pontos que variam entre 1 ("não ou raramente”) e 5 ("sempre”); o último ponto de ancoragem considera a situação "não sei”. A escala possui bons indicadores de validade construto e de critério.

Spiritual Intelligence Self-Report Inventory (SISRI-24; King, 2008; King \& DeCicco, 2009). É uma medida de auto-relato da inteligência espiritual com 24 itens. Inclui quatro subescalas: pensamento crítico existencial (PCE) (i.e, a capacidade de pensar criticamente questões existenciais como vida, morte, realidade e existência), medida por 7 itens $(\alpha=.85)$; produção de sentido pessoal (PSP) (i.e., a capacidade atribuir sentido e propósito a todas as experiências físicas e mentais), medida por 5 itens $(\alpha=.84)$; consciência transcendental (CT) (i.e., a capacidade de perceber as dimensões transcendentes do self, dos outros e do mundo físico), medida por 7 itens ( $\alpha$ = .89); e expansão do estado consciente (EEC) (i.e., a capacidade de aceder a estados expandidos ou espirituais de consciência por vontade própria), medida por 5 itens $(\alpha=.92)$. As respostas aos itens variam numa escala de tipo Likert com 5 pontos entre 0 ("nada verdadeiro") e 4 (“completa mente verdadeiro"), com respostas mais elevadas a representarem níveis mais elevados do construto. É possível calcular um resultado total de inteligência espiritual, a variar entre 0 e 96, somando todos os itens. King e DeCicco (2009) obtiveram evidências que apoiam a fidelidade teste-reteste e a validade de construto e de critério do SISRI-24.

Positive and Negative Affect Schedule (PANAS; Watson, Clark, \& Telleguen, 1988). É uma medida de auto-relato com duas subescalas que proporcionam duas medidas breves de afecto positivo $(\alpha=.87)$ e de afecto negativo ( $\alpha=.87$ ). Os itens consistem em adjectivos que representam estados de humor relacionados com afectos positivos/negativos. Os respondentes assinalam em que medida têm sentido cada emoção durante um período de tempo específico ("últimas semanas”) usando uma escala de tipo Likert com 5 pontos de ancoragem variando entre 1 (“minimamente") e 5 ("totalmente"). Calcula-se um valor por subescala, somando os respectivos itens, que varia entre 10 e 50. A PANAS apresenta indicadores adequados de validade de construto e de critério.

Rosenberg Self-Esteem Scale(RSES; Rosenberg, 1965). É uma medida unidimensional composta por 10 itens que consistem em afirmações de autoestima e autoaceitação a que os participantes respondem com base numa escala de tipo Likert com 4 pontos que variam entre 1 ("discordo muito”) e 4 (“concordo muito”). Metade dos itens têm formulação negativa, requerendo que as respostas sejam revertidas antes do cálculo do valor global da escala que pode variar entre 10 e 44; valores elevados correspondem a níveis elevados do construto. Os valores de fidelidade têm-se revelado consistentemente superiores a .85.

Future Time Perspective Scale (FTPS; Husman \& Shell, 2008). É uma medida de auto-relato que visa medir aspectos relacionados com a perspectiva temporal global, 
através de 27 itens que se repartem em quatro subescalas: instrumentalidade, medida por 12 itens, dos quais $8 \mathrm{com}$ formulação negativa ( $\alpha=.82)$; valência, medida por 7 itens, 1 dois quais em formulação negativa $(\alpha=.72)$; velocidade, medida com 3 itens, todos eles negativamente formulados ( $\alpha=.72$ ); extensão, medida com 5 itens, dos quais 3 com formulação negativa $(\alpha=.74)$. A escala possui indicadores adequados de validade de construto e divergente.

Meaning in Life Questionnaire (MLQ; Steger, Frazier, Oishi, \& Kaler, 2006). É uma medida de auto-relato do significado pessoal com 10 itens. Inclui duas subescalas: presença, medida com 5 itens, um dos quais formulado negativamente $(\alpha=.86)$; procura, avaliada com 5 itens ( $\alpha$ $=.87$ ). As respostas aos itens variam numa escala de tipo Likert com 7 pontos, entre 1 (“absolutamente falso”) e 7 (“absolutamente verdadeiro"); valores elevados nas duas subescalas representam níveis elevados de presença ou de procura de sentido para a vida. Os autores obtiveram evidências de adequada estabilidade temporal, bem como bons indicadores de validade convergente e discriminante nas duas subescalas.

\section{Procedimento}

O estudo foi realizado durante o segundo semestre do presente ano lectivo, em contexto de sala de aula, com uma duração aproximada de 25 minutos. Assegurou-se o consentimento informado dos participantes, tendo sido garantida a confidencialidade e o direito de abandono a qualquer momento sem penalização.

\section{Resultados}

As análises de Rasch proporcionam indicadores que permitem quantificar o ajustamento do modelo, estimar os parâmetros do item e da pessoa e diagnosticar o funcionamento das categorias de resposta aos itens (Fox \& Jones, 1998). A Tabela 1 apresenta as estatísticas dos itens para o ajustamento (infit e outfit), a localização $\left(D_{i}\right)$ e o erro padrão $(E P)$ que permitem avaliar a validade de conteúdo da versão portuguesa da SET (SET-P), bem como as respectivas média $(M)$ e desvio padrão $(D P)$ para além dos coeficientes de validade estrutural (CE) da escala (Wolfe \& Smith, 2007).

Em termos de validade de conteúdo, a Tabela 1 permite constatar que os valores médios de infit e outfit igualam ou praticamente coincidem com o valor esperado de 1.0 que indica um ajustamento perfeito dos itens; todos os 11 itens da escala revelam valores individuais dentro do intervalo [.5 - 1.5] que Wright e Linacre (1994) estipulam como produtivo para a medida, facto que indicia a inexistência de itens redundantes e a existência de homogeneidade entre os itens da SET-P. Este indicador da unidimensionalidade é confirmado por outros estimadores da modelação Rasch, com o fim de analisar o contributo dos itens na definição de um construto central para a estrutura interna da escala. De facto, as correlações ponto -medida $\left(r_{p m}\right)$, similares às correlações item-total da teoria clássica dos testes (TCT), com valores entre .56 e .79 sugerem a inexistência de ruído não modelado ou de dependência nos dados (Linacre, 2011), permitindo concluir que cada um dos itens contribui para definir um mesmo construto comum (e.g., pensamento existencial); para além disso, os valores de erro-padrão dos itens, entre .08 e .09, também indicam que a fidelidade dos itens é elevada. De facto, o valor de item separation reliability (.98), excelente (Fisher, 2007), permite concluir que os itens da SET-P foram medidos com elevada precisão.

Tabela 1.

Propriedades psicométricas da SET-P

\begin{tabular}{crrrrrr}
\hline & \multicolumn{2}{c}{ MNSQ } & & & & \\
\cline { 2 - 4 } Item & Infit & Outfit & \multicolumn{1}{c}{$D_{i}$} & $E P$ & $r_{p m}$ & CE \\
\hline 1 & 1.09 & 1.12 & -.94 & .09 & .56 & -.41 \\
2 & 1.14 & 1.12 & -.45 & .08 & .64 & -.62 \\
3 & 1.06 & 1.00 & .37 & .08 & .75 & .50 \\
4 & 1.25 & 1.21 & -.04 & 08 & .67 & .35 \\
5 & .90 & .90 & -.75 & .08 & .65 & -.41 \\
6 & 1.40 & 1.31 & 1.00 & .09 & .69 & .12 \\
7 & .76 & .80 & .61 & .09 & .78 & .63 \\
8 & 1.02 & 1.06 & .67 & .09 & .77 & .26 \\
9 & .86 & .86 & .00 & .08 & .76 & -.38 \\
10 & .61 & .63 & -.33 & .08 & .79 & -.11 \\
11 & .84 & .85 & -.15 & .08 & .76 & .11 \\
\hline M & 1.00 & .99 & .00 & .08 & & \\
DP & .22 & .19 & .58 & .00 & --- & -- \\
\hline
\end{tabular}

Com o propósito de averiguar a validade estrutural da escala, calculou-se a Análise de Componentes Principais (ACP) dos resíduos padronizados depois de controlar a dimensão Rasch, para determinar se os itens da escala correspondem ao construto definido (Smith, 2004). Perante a falta de consenso nos critérios indicadores de uma dimensão secundária (Chou \& Wang, 2010), os autores optaram por considerar um eigenvalue inferior a 2.0 e uma variância explicada pelo primeiro componente dos resíduos não superior a $10 \%$ como indicadores da unidimensionalidade da SET-P (Linacre, 2011). A última coluna da Tabela 1, relativa aos coeficientes estruturais dos 11 itens da SET-P, permite constatar que 5 deles têm valores superiores ao ponto de corte (.40), sendo que apenas os itens 2 e 7 excedem ligeiramente o limiar de .60; para além disso, o primeiro componente possui um eigenvalue $=1.70$ que representa uma variância residual de $6.7 \%$, considerada boa segundo Fisher (2007), o que sugere que os resíduos padronizados não têm informação sistemática adicional. Uma vez que a variância explicada pela medida (57.4\%) a classifica como uma dimensão de medida forte (Reckase, 1979), estes resultados indicam a unidimensionalidade da SET-P, pressuposto requerido pelo modelo de Rasch.

A validade substantiva refere-se ao diagnóstico do funcionamento empírico das categorias da escala de resposta, com o propósito de determinar se estas funcionam em conformidade com aquilo que era esperado pelo autor do instrumento quando desenvolveu os respectivos itens (Wolfe \& Smith, 2007). No contexto do RSM, Linacre (2002) propôs um conjunto de critérios para determinar a eficiência das categorias de resposta: (a) distribuição uniforme das frequências das respostas pelas diferentes categorias, com um mínimo de 10 observações em cada uma; (b) progressão monotónica da medida média obser- 
vada $-B_{n}$ - e das calibrações dos passos $-F_{k}$ - ao longo das categorias de resposta; e (c) outfit MNSQ, mais sensível que o infit a respostas não esperadas, das categorias de resposta inferior a 2.0. A Tabela 2 resume as estatísticas necessárias para avaliar em que medida as 6 categorias de resposta da SET-P cumprem com estes critérios. A sua análise permite concluir que a estrutura da escala de resposta cumpre com os critérios estipulados por Linacre (2002), uma vez que cada uma das categorias possui uma frequência observada de respostas superior a 10 e um valor de outfit inferior a 2.0; para além disso, as medidas médias observadas e a calibração dos passos aumentam de forma monotónica ao longo das 6 categorias de resposta.

Tabela 1.

SET: estatísticas das categorias de resposta

\begin{tabular}{cccccccc}
\hline & \multicolumn{3}{c}{ Observada } & & \multicolumn{2}{c}{ MNSQ } & \\
\cline { 2 - 3 } Categoria & $f_{i}$ & $\%$ & $B_{n}$ & Infit & Outfit & $F_{k}$ \\
\hline 1 & 232 & 11 & -2.35 & .94 & .95 & --- \\
2 & 549 & 25 & -1.61 & .93 & .95 & -2.80 \\
3 & 558 & 25 & -.88 & .93 & .94 & -1.27 \\
4 & 510 & 23 & .00 & .84 & .84 & -.42 \\
5 & 309 & 14 & .85 & 1.11 & 1.10 & .87 \\
6 & 42 & 2 & 2.11 & 2.07 & 1.65 & 3.62 \\
\hline
\end{tabular}

E adequação da escala de resposta é corroborada pelo resultado da análise feita ao ajustamento das pessoas; a média e o desvio padrão para as estatísticas de ajustamento foram .99 e .67 (infit), respectivamente, e .99 e .66 (outfit), respectivamente. Uma vez que a percentagem de pessoas com infit e/ou outfit superior a 1.5, valor do limiar superior de aceitabilidade psicométrica para a modelação Rasch (Wright \& Linacre, 1994), é baixa (16.0\%), pode-se afirmar que o ajuste ao modelo é adequado (Fisher, 2007). De facto, para a maioria das pessoas, os parâmetros foram estimados com elevada precisão; o valor do person separation reliability (.89), estimador de fidelidade semelhante ao alfa de Cronbach que mede a proporção de variância da pessoa que não é explicada pelo erro de medida, confirma-o. A SET-P permite, assim, discriminar a amostra em três níveis quanto ao atributo medido.

A Figura 1 apresenta a representação conjunta pessoa-item na mesma escala métrica comum (i.e., logits). Os 11 itens da SET-P encontram-se organizados por ordem decrescente de dificuldade, operacionalizada pela quantidade do atributo medido na pessoa. Assim, o item 6 é aquele que representa a maior dificuldade de escolha, enquanto o item 1 é o mais fácil de subscrever.

É expectável que as estimativas relativas ao atributo da pessoa e à dificuldade do item se sobreponham, de forma substancial, para que o conjunto dos itens ser apropriado para a amostra de sujeitos. Sempre que a diferença entre as médias destas estimativas é inferior a 1 logit (Bond \& Fox, 2007), significa que a informação contida nos itens permite discriminar as pessoas de forma muito precisa no construto que o instrumento se propõe medir. No caso do presente estudo, o valor dessa diferença (-.71) sugere um nível de pensamento existencial médio baixo, o que explica que a maior parte dos sujeitos se esteja abaixo do ponto da variável em que os itens se situam. Em consequência, uma vez que a média dos sujeitos no atributo é baixa (i.e., dificuldade elevada dos itens), correspondendo a indivíduos com pensamento existencial médio ( \pm 1 logit) que tendem a coincidir com os itens da escala, estes não permitem medir com elevada precisão as pessoas com nível de pensamento existencial baixo (cf. Figura 1).

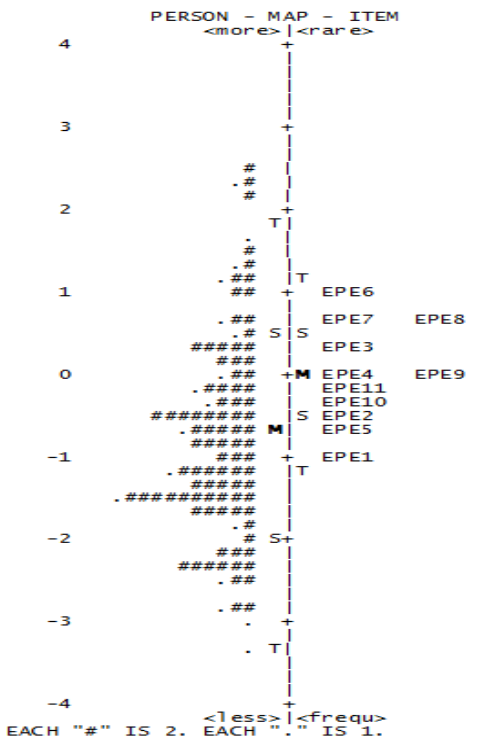

Figura 1. Representação conjunta de pessoas e itens

Foi ainda analisado o funcionamento diferencial dos itens (DIF) para avaliar a validade dos resultados da SET-P relativamente ao género. Foi calculada a diferença padronizada entre as localizações dos parâmetros masculino e feminino, depois de se terem ajustado as possíveis diferenças relativas ao sexo na distribuição do pensamento existencial; usou-se o procedimento de Bonferroni que corrigiu o nível de significância em função do número de comparações (.05/11) (Linacre, 2011). Seguindo este critério conservador, nenhum dos itens da SET-P revelou localização superior a .50, valor estipulado por Wright \& Douglas (1975) como ponto de corte para o contraste DIF, nem no sexo masculino, nem no sexo feminino.

A SET-P correlacionou a um nível estatisticamente significante com a inteligência espiritual ( $r=.65)$, o afecto positivo $(r=.29)$, com a procura e a presença de sentido para a vida ( $r=.25$ e $r=.22$, respectivamente) e com a extensão da PTF $(r=.15)$.

\section{Discussão}

Globalmente, o estudo confirma que a SET-P é um instrumento válido na avaliação do pensamento existencial dos alunos do ensino superior. Os resultados validam a tese da unidimensionalidade da escala defendida pelos autores (Allan \& Shearer, 2012); a utilização do modelo de Rasch, permite que os níveis de pensamento existencial das pessoas e de dificuldade com que os itens da SET-P medem este construto sejam hierarquizados num mesmo contínuo escalar. Fá-lo em ambos os casos com elevado grau de precisão, provando assim a adequação da escala de resposta com seis alternativas, e sem enviesamento quanto ao género de pertença.

A principal implicação deste estudo resulta, tal como os resultados comprovam, na demonstração da SET-P é uma medida intervalar, não ordinal. O ajustamento dos 
dados ao modelo de Rasch permite criar uma escala linear (logits) para itens e pessoas que proporciona valores expressos nas mesmas unidades. Ao demonstrar-se que os itens da SET-P e a amostra usada na sua calibração são independentes, permitindo comparar a localização do atributo do sujeito na variável latente directamente com o nível de dificuldade do item usado para medir esse atributo, confirma-se a existência de uma estrutura simples que é essencial na medição invariante (Engelhard, 2008), base para modelos de medida úteis (Rasch, 1960). A natureza intervalar da medida traduz-se em vantagens concretizadas na utilização válida de testes paramétricos, o que até aqui não era possível afirmar, justificada pela invariância da medida (pessoas/itens).

O estudo apresenta algumas limitações que importa superar numa próxima investigação, designadamente no que diz respeito à amostra que, embora reproduzisse as características demográficas da população visada, não é representativa da realidade Portuguesa. Para que os resultados possam ser generalizáveis importa que no futuro se recorra a métodos de amostragem aleatória.

\section{Referências}

Allan, B.A., \& Shearer, C.B. (2012). The Scale for Existential Thinking. International Journal of Transpersonal Studies, 31(1), 21-37.

Bond, T.G., \& Fox, C.M. (2007). Applying the Rasch model. Fundamental measurement in the human sciences ( $2^{\text {nd }}$ ed.). Mahwah, NJ: Lawrence Erlbaum.

Chou, Y.-T., \& Wang, W.-C. (2010). Checking dimensionality in item response models with principal component analysis on standardized residual. Educational and Psychological Measurement, 70, 717-731. http:// dx.doi.org/10.1177/0013164410379322

Engelhard, G. (2008). Historical perspectives on invariant measurement: Guttman, Rasch, and Mokken. Measument, 6, 155-189. http://dx.doi.org/10.1080/1536636 0802197792

Fisher, W.P. Jr. (2007). Rating scale instrument quality criteria. Rasch Measurement Transactions, 21, p. 1095.

Fox, C.M., \& Jones, J.A. (1998). Uses of Rasch modeling in counseling psychology research. Journal of Counseling Psychology, 45, 30-45. http://dx.doi.org/10.1037// 0022-0167.45.1.30

Gardner, H. (2004). Frames of mind: The theory of multiple intelligences. New York, NY: Basic Books.

Husman, J., \& Shell, D.F. (2008). Beliefs and perceptions about the future: A measurement of future time pers pective. Learning and Individual Differences, 18(2), 166-175.http://dx.doi.org/10.1016/j.lindif.2007.08.001

King, D.B. (2008). Rethinking claims of spiritual intelligence: A definition, model, and measure. Unpublished master's thesis, Trent University, Peterborough, ON, Canada.

King, D.B., \& DeCicco, T.L. (2009). A viable model and self-report measure of spiritual intelligence. International Journal of Transpersonal Studies, 28(1), 68-85.

Linacre, J.M. (2002). Optimizing rating scale category effectiveness. Journal of Applied Measurement, 3(1), 85-106.
Linacre, J.M. (2011). Winsteps Rasch measurement computer program, version 3.73.0. [Computer program] Chicago, Il: Winsteps.com.

Messick, S. (1995). Validity of psychological assessment: Validation of inferences from persons' responses and performances as scientific inquiry into score meaning. American Psychologist, 50, 741-749. http://dx.doi.org/10.1037//0003-066x.50.9.741

Rasch, G. (1960). Probabilistic models for some intelligence and attainment tests. Copenhagen: The Danish Institute of Educational Research. [Expanded edition, 1980]. Chicago: University of Chicago Press.]

Reckase, M. (1979). Unifactor latent trait models applied to multifactor tests: results and implications. Journal of Educational Statistics, 4, 207-230. http://dx.doi.org/10 .2307/1164671

Rosenberg, M. (1965). Society and adolescence self-image. Princeton, NJ: Princeton University Press. http://dx.doi.org/10.1037/t01038-000

Shearer, C.B. (2006). Development and validation of a scale for existential thinking. Unpublished manuscript. Kent State University, $\mathrm{OH}$.

Smith Jr, E.V. (2004). Detecting and evaluating the impact of multidimensionality using item fit statistics and principal component analysis of residuals. In: E.V. Smith Jr \& R.M. Smith (Eds.), Introduction to Rasch measurement (pp. 575-600). Maple Grove, MN: JAM Press.

Spinelli, E. (2005). The interpreted world: An introduction to phenomenological psychology. Thousand Oaks, CA: SAGE.

Steger, M.F., Frazier, P., Oishi S., \& Kaler, M, (2006). The Meaning in Life Questionnaire: Assessing the presence of and the search for meaning in life. Journal of Counseling Psychology, 53(1), 80-93. http://dx.doi. org/10.1037/0022-0167.53.1.80

Watson, D, Clark, L.A., \& Tellegen, A. (1988). Development and validation of brief measures of positive and negative affect: The PANAS scales. Journal of Perso nality and Social Psychology, 6, 1063-1070. http://dx. doi.org/10.1037/t13775-000

Wolfe, E.W., \& Smith Jr, E.V. (2007). Instrument development tools and activities for measure validation using Rasch models: Part II - Validation activities. In In E.V. Smith Jr \& R.M. Smith (Eds.), Rasch measurement: Advanced and specialized applications (pp. 243-290). Maple Grove, MN: JAM Press.

Wright, B.D., \& Douglas, G.A. (1975). A better procedure for sample-free item analysis. Research Memorandum. Statistical Laboratory. Department of Education. University of Chicago.

Wright, BD., \& Linacre, J.M. (1994). Reasonable mean-square fit values. Rasch Measurement Transactions, 8, p. 370.

Wright, B.D., \& Masters, G.N. (1982). Rating scale analysis. Chicago, Il: MESA Press.

Wright, B.D., \& Mok, M.M. (2004). An overview of the family of Rasch measurement models. In E.V. Smith Jr \& R.M. Smith (Eds.), Introduction to Rasch measurement (pp. 1-24). Maple Grove, MN: JAM Press. 